\title{
КОДИРОВАНИЕ ДАННЫХ РАЗВЕДКИ ТОРФЯНЫХ МЕСТОРОЖДЕНИЙ ДЛЯ ПОДСЧЕТА И ОЦЕНКИ ЗАПАСОВ НА ЭВМ
}

В статье излагается методика кодирования исходной информации для автоматизированной системы (AC) подсчета и оценки запасов торфа, создание которой необходимо для облегчения камеральной обработки геологоразєедочных материалов. Составленная по этой методике программа ввода исходных данных на магнитную ленту ЭВМ «Минск-32» реализована на языке ЯСК в вычислительном центре Эстонекого республиканского союза потребительских обществ.

Подлежащие обработке с помощью АС материалы геологоразведочных работ на торф содержат данные зондирования, стратиграфического бурения, опробования, исследования пнистости и оконтуривания торфяной залежи (Инструкция по разведке торфяных месторождений, 1973; Методическое руководство по типологическому дешифрированию торфяных месторождений, 1967). Для характеристики расположения пунктов этой информации на плоскость проецируется поверхность исследуемого месторождения. Положение точки на плоскости определяется декартовой системой координат. В целях более простого использования АС предполагается, что все точки находятся в первой четверти системы координат. Используемая шифровка классификации болотных отложений приведена в приложении.

Подготовка исходных данных для перфорации начинается с раздела массива на логически цельные части - т. н. элементы (Кушнерев и др., 1973). Первое число каждого элемента - это количество чисел в элементе, которое не должно превышать 320 . Для контроля все числа массива еще раз делятся на группы с количеством чисел до 480 , по которым вычисляются суммы. В один контрольный элемент может входить целое число элементов или, наоборот, в одном элементе может быть целое число контрольных элементов. Для удобства дальнейшей обработки исходной инфіормации устанавливаются, в зависимости от вида данных, конкретные ограничения на количество чисел как в элементе, так и в контрольном элементе.

При кодировании результатов зондирования и стратиграфического бурения за элемент принимают параметр поперечника или данные бурения в одном пункте. Структура элементов приведена в табл. 1.

Параметр поперечника должен быть первым элементом информации поперечника. Остальные элементы в массиве упорядочивают по возрастанию номера поперечника и пикета. Например, наблюдаем пункты бурения $\left(a_{i}, b_{i j}\right)$ и $\left(a_{k}, b_{k l}\right)$. Если из двух условий $\left(a_{i}<a_{k}\right.$ или $a_{i}=a_{k}$, $\left.b_{i j}<b_{k l}\right)$ выполняется одно, тогда элемент, соответствующий информации в пункте $\left(a_{i}, b_{i j}\right)$, предшествует элементу, соответствующему информации 


\section{Структура элементов зондирования и стратиграфического бурения}

Параметр поперечника пунктов зондирования

Параметр поперечника пунктов стратиграфического бурения

Информация пункта зондирования

Информация пункта стратиграфическо-

го бурения с определением вида залежи

Информация пункта стратиграфического бурения без определення вида залежи

$$
\begin{aligned}
& n_{i j}, a_{i},-1, x_{i}, y_{i}, d_{i}, \alpha_{i} \\
& n_{i j}, a_{i},-1,0, x_{i}, y_{i}, d_{i}, \alpha_{i} \\
& n_{i j}, a_{i}, b_{i j}, p_{i j 1}, s_{i j 1}, \ldots, p_{i j k}, s_{i j k} \\
& n_{i j}, a_{i}, b_{i j}, l_{i j}, p_{i j 1}, s_{i j 1}, \ldots, p_{i j k}, s_{i j k} \\
& n_{i j}, a_{i}, b_{i j}, 0, p_{i j 1}, s_{i j 1}, \ldots, p_{i j k}, s_{i j k}
\end{aligned}
$$

О бозначения:

$n_{i j}$ - количество чисел в элементе;

$a_{i}$ - номер поперечника;

$b_{i j}$ - номер пикета на поперечнике $a_{i}$;

$\left(a_{i}, b_{i j}\right)$ - пункт бурения на поперечнике $a_{i}$, пикета $b_{i j}$;

$\left(x_{i}, y_{i}\right)$ - координаты начального пункта бурения на поперечнике $a_{i}$;

$d_{i}$ - среднее расстояние между пунктами бурения на поперечнике $a_{i}$;

$\alpha_{i}$ - угол подъема поперечника $a_{i}$ в градусной мере;

$p_{i j k}-$ глубина $k$-го слоя в пункте бурения $\left(a_{i}, b_{i j}\right)$;

$s_{i j k}-$ шифр $\kappa$-го слоя (прил., кл. 1) в пункте бурения $\left(a_{i}, b_{i j}\right)$;

$l_{i j}$ - шифр вида залежи (прил., кл. 3) в пункте бурения $\left(a_{i}, b_{i j}\right)$.

Таблица 2

Пример записи результатов специального стратиграфического бурения, подлежащих перфорации

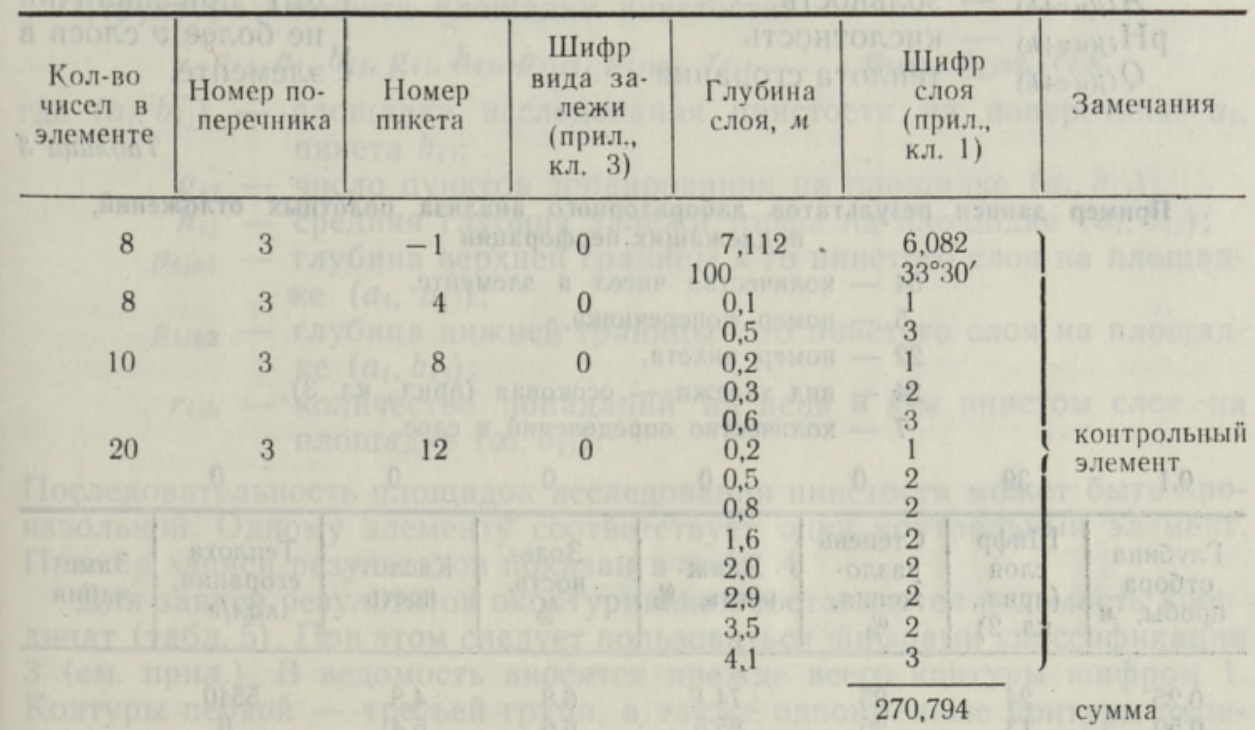

в пункте $\left(a_{k}, b_{k l}\right)$. Нумерация пикетов должна начинаться с одной стороны поперечников. Так как в имеющихся программах сортировка исходной информации не реализована, ее выполняют перед кодированием. Избежать переписывания позволяют карты краевой перфорации. 
В контрольном элементе должно быть целое число элементов. При вычислении контрольных сумм угол подъема $\alpha$ рассматривается как реальное число, где градусы составляют целое, минуты и секунды - дробную часть.

Табл. 2 иллюстрирует пример записи результатов специального стратиграфического бурения. Для данных зондирования можно пользоваться этой же формой, но без графы шифра вида залежи.

При кодировании результатов лабораторного анализа болотных отложений за элемент принимают информацию одного пункта опробования. При составлении элемента следует следить за условием $n_{i j} \leqslant 320$. Если эта информация не помещается в один элемент, ее разбивают на два элемента с таким расчетом, чтобы первый включал максимальное количество информации, но без разделения информации слоя между элементами.

Структура элемента пункта опробования:

$$
\begin{gathered}
n_{i j}, a_{i}, b_{i j}, l_{i j}, m_{i j}, p_{i j(u v+1)}, s_{i j(u v+1)}, R_{i j(u v+1)}, \\
W_{i j(u v+1)}, A_{i j(u v+1)}, \mathrm{pH}_{i j(u v+1)}, Q_{i j(u v+1)}, p_{i j(u v+2),}, \\
s_{i j(u v+2)}, R_{i j(u v+2)}, W_{i j(u v+2)}, A_{i j(u v+2)}, \mathrm{pH}_{i j(u v+2),}, p_{i j(u v+k)}, s_{i j(u v+k)}, R_{i j(u v+k)}, W_{i j(u v+k)}, \\
Q_{i j(u v+2)}, \ldots, p_{i j(u v+k)}, \mathrm{pH}_{i j(u v+k)}, Q_{i j(u v+k)},
\end{gathered}
$$

\begin{tabular}{|c|c|c|}
\hline$p_{i j(u v+k)}$ & - глубина (без мощности очеса) & $k$-го слоя в $u$-м \\
\hline$s_{i j(u v+k)}$ & - шифр (прил., кл. 2) & (начиная с нуля) \\
\hline$R_{i j(u v+k)}$ & - степень разложения & элементе пункта \\
\hline$W_{i j(u v+k)}$ & влажность & опробования $\left(a_{i}\right.$, \\
\hline$A_{i j(u v+k)}$ & зольность & $\left.b_{i j}\right)$ при наличии \\
\hline $\mathrm{H}_{i j(u v+k)}$ & кислотность & не более $v$ слоев в \\
\hline$Q_{i j(u v+k)}$ & - теплота сгорания & элементе. \\
\hline
\end{tabular}

где $m_{i j}-$ количество определений в пункте опробования $\left(a_{i}, b_{i j}\right)$;

Таблица 3

Пример записи результатов лабораторного анализа болотных отложений, подлежащих перфорации

54 - количество чисел в элементе,

5 - номер поперечника,

22 - номер пикета,

24 - вид залежи - осоковая (прил., кл. 3),

7 - количество определений в слое.

0,1

39

0

0

0

0

\begin{tabular}{c|c|c|c|c|c|c|c}
\hline $\begin{array}{c}\text { Глубина } \\
\text { отбора } \\
\text { пробы, }\end{array}$ & $\begin{array}{c}\text { Шифр } \\
\text { слоя } \\
\text { (прил., } \\
\text { кл. 2) }\end{array}$ & $\begin{array}{c}\text { Степень } \\
\text { разло- } \\
\text { жения, } \\
\%\end{array}$ & $\begin{array}{c}\text { Влаж- } \\
\text { ность, } \%\end{array}$ & $\begin{array}{c}\text { Золь- } \\
\text { ность, } \\
\%\end{array}$ & $\begin{array}{c}\text { Кислот- } \\
\text { ность }\end{array}$ & $\begin{array}{c}\text { Теплота } \\
\text { сгорания, } \\
\text { кал/2 }\end{array}$ & $\begin{array}{c}\text { Заме- } \\
\text { чания }\end{array}$ \\
\hline
\end{tabular}

\begin{tabular}{llllllc}
0,25 & 24 & 25 & 74,1 & 6,8 & 4,8 & 5540 \\
0,50 & 13 & 25 & 85,5 & 6,6 & 5,4 & 0 \\
0,75 & 13 & 25 & 86,8 & 7,2 & 5,4 & 5550 \\
1,00 & 13 & 30 & 86,5 & 7,3 & 5,2 & 0 \\
1,25 & 13 & 30 & 86,4 & 7,1 & 5,2 & 5620 \\
1,60 & 13 & 30 & 86,0 & 11,8 & 5,3 & 0 \\
\cline { 3 - 5 } & & & & & & 17703,85
\end{tabular}


Пример записи результатов исследования пнистости, подлежащих перфорации

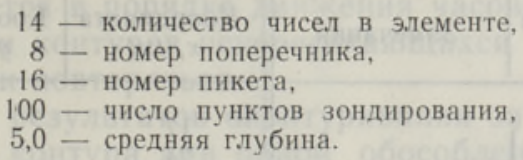

\begin{tabular}{c|c|c|c}
\hline $\begin{array}{c}\text { Губина верх- } \\
\text { ней границы } \\
\text { слоя, } M\end{array}$ & $\begin{array}{c}\text { Глубина ниж- } \\
\text { ней границы } \\
\text { слоя, } M\end{array}$ & $\begin{array}{c}\text { Кол-во попада- } \\
\text { ний на пень в } \\
\text { слое }\end{array}$ & 3амечания \\
\hline
\end{tabular}

\begin{tabular}{ccc}
0,2 & 1,5 & 5 \\
2,4 & 3,1 & 26 \\
3,6 & 4,6 & 8 \\
\cline { 2 - 2 } & & 197,4
\end{tabular}

сумма

Последовательность пунктов опробования значения не имеет. Одному элементу соответствует один контрольный элемент. Пример записи результатов анализа приведен в табл. 3. Данные очеса или минерального наноса записываются над графами. Если какие-то анализы выполнены реже других, их результаты записываются на строчке верхнего уровня слоя, а строчки по нижеследующим уровням заполняются нулями. В случае отсутствия анализов также проставляются нули.

При кодировании результатов исследования пнистости за элемент принимают информацию одной площадки. При этом предполагается, что пнистые слои предварительно выделены вручную по методике Ларгина (Методическое руководство по камеральной обработке материалов разведки торфяных месторождений, 1969).

Структура элемента площадки пнистости:

$$
n_{i j}, a_{i}, b_{i j}, g_{i j}, h_{i j}, p_{i j 11}, p_{i j 12}, r_{i j 1}, \ldots, p_{i j h 1}, p_{i j k 2}, r_{i j h},
$$

где $\left(a_{i}, b_{i j}\right)$ - площадка исследования пнистости на поперечнике $a_{i}$, пикета $b_{i j}$;

$g_{i j}$ - число пунктов зондирования на площадке $\left(a_{i}, b_{i j}\right)$;

$h_{i j}-$ средняя глубина залежи торфа на площадке $\left(a_{i}, b_{i j}\right)$;

$p_{i j h 1}$ - глубина верхней границы $k$-го пнистого слоя на площадке $\left(a_{i}, b_{i j}\right)$;

$p_{i j k 2}$ - глубина нижней границы $k$-го пнистого слоя на площадке $\left(a_{i}, b_{i j}\right)$;

$r_{i j h}$ - количество попаданий на пень в $k$-м пнистом слое на площадке $\left(a_{i}, b_{i j}\right)$.

Последовательность площадок исследования пнистости может быть произвольной. Одному элементу соответствует один контрольный элемент. Пример записи результатов показан в табл. 4 .

Для записи результатов оконтуривания составляется ведомость координат (табл. 5). При этом следует пользоваться шифрами классификации 3 (см. прил.). В ведомость вносятся прежде всего контуры шифром 1. Контуры первой - третьей групп, а также одноименные контуры записываются последовательно. Направление движения на плане - по часовой стрелке.

Выделение контуров непересекающихся участков (первой и второй или первой и третьей групп) определяется их расположением относительно друг друга. При этом возможны три случая. 
Пример записи результатов оконтуривания, подлежащих перфорации

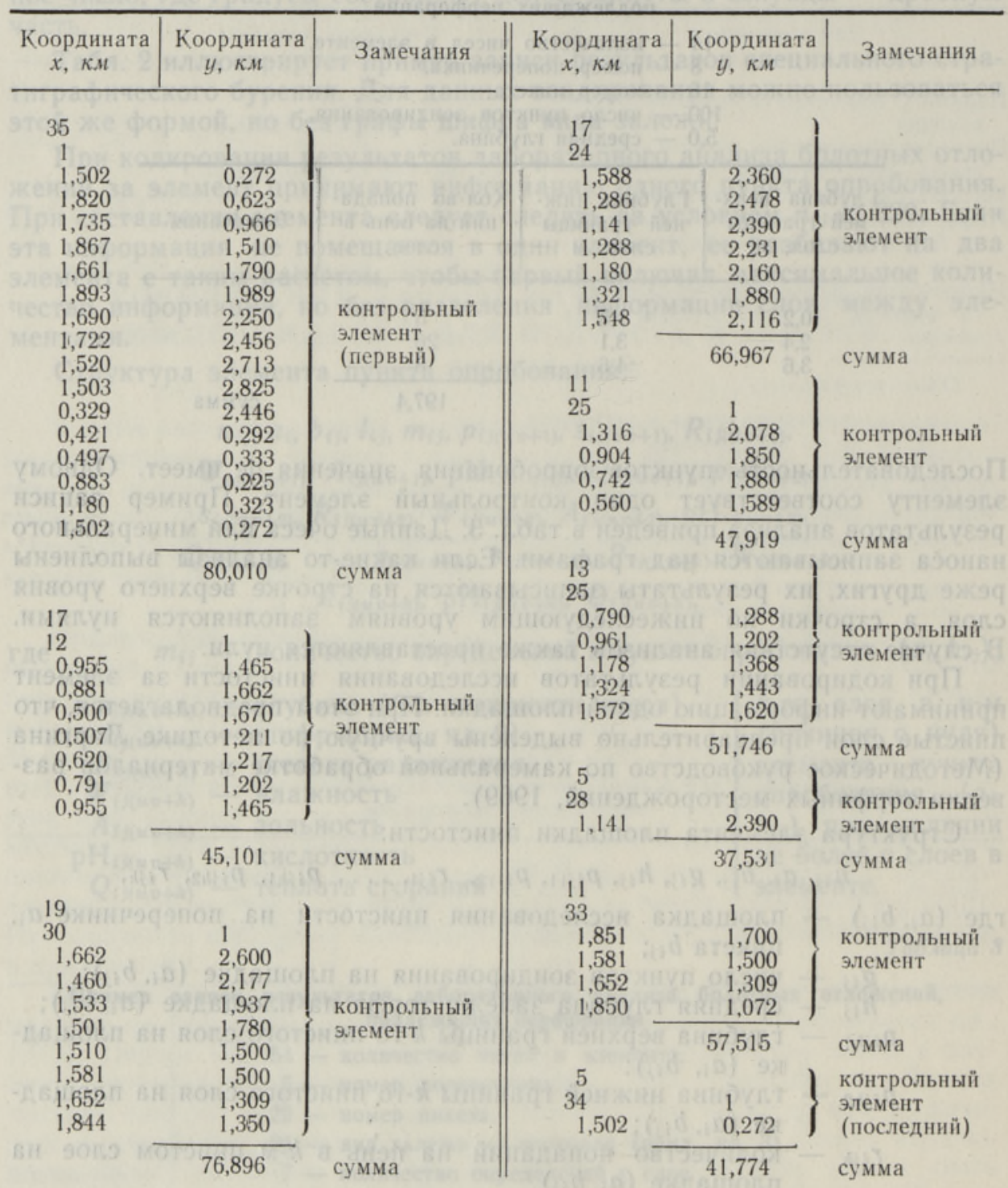

1. Контур выделяемого участка не имеет ни одного совпадения с контурами ранее выделенных участков. Пункты контура образуют замкнутый многоугольник. Признак замкнутости - совпадение координат первого и последнего пунктов.

2. Контур выделяемого участка совпадает с контурами ранее выделенных участков в одном месте. В этом случае пункты контура, подлежащие перфорации, образуют ломаную линию, крайние отрезки которой должны пересекаться с уже выделенными контурами по меньшей мере один раз, а с одним и тем же контуром не более одного раза. За первый пункт ломаной линии принимается тот ее крайний пункт, от которого можно двигаться вдоль этой линии по часовой стрелке. 
3. Контур выделяемого участка совпадает с контурами ранее выделенных участков в нескольких местах. Пункты контура этого участка, подлежащие перфорации, образуют обособленные ломаные линии, запись которых осуществляется в порядке движения часовой стрелки.

В ведомости среди контуров непересекающихся участков один и тот же отрезок не должен повторяться.

При кодировании результатов оконтуривания за элемент принимают информацию одного контура или одной обособленной части контура. Если эта информация не помещается в один элемент, ее делят между двумя или более элементами с таким расчетом, чтобы информация одного пункта находилась в одном элементе.

Структура элементов контура такова: 1) информация последнего элемента замкнутого контура:

$$
n_{i j}, s_{i}, t_{i j}, x_{i j(u v+1)}, y_{i j(u v+1)}, \ldots, x_{i j(u v+k)}, y_{i j(u v+k)}, x_{i j 1}, y_{i j 1} ;
$$

2) информация прочих элементов:

$$
n_{i j}, s_{i}, t_{i j}, x_{i j(u v+1)}, y_{i j(u v+1)}, \ldots, x_{i j(u v+k)}, y_{i j(u v+k)} \text {. }
$$

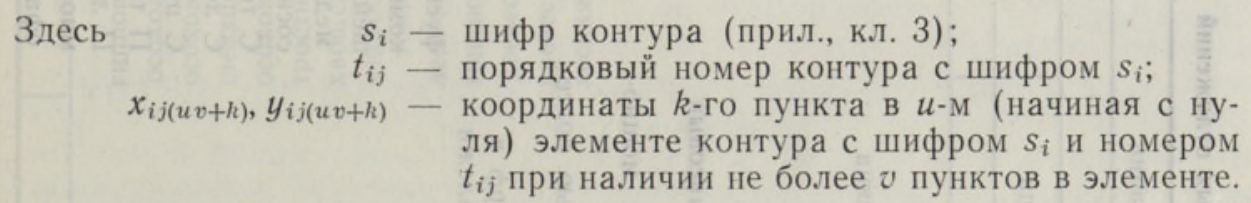

Если контур не помещается в один элемент, то $s_{i}$ и $t_{i j}$ во всех элементах, кроме первого, замещаются нулями.

В случае полного совпадения границы участка с контурами ранее выделенных участков информация элемента этого участка имеет структуру:

$$
n_{i j}, s_{i}, t_{i j}, x_{i j}, y_{i j} .
$$

Координаты единственного пункта в элементе должны быть представлены среди координат ранее выделенных контуров, образующих границу этого участка. Такой элемент встречается во второй и третьей группах контуров. Наличие его является признаком конца информации о контурах в группе. Одному элементу соответствует один контрольный элемент.

\section{ЛИТЕРА Т У Р А}

Инструкция по разведке торфяных месторождений. 1973. М.

К уш н ерев Н. Т., Н ем ен м ан М. Е., Ц а гель с кий В. И. 1973. Программирование для ЭВМ «Минск-32». М.

Методическое руководство по камеральной обработке материалов разведки торфяных месторождений. 1969. М.

Методическое руководство по типологическому дешифрированию торфяных месторож. дений. 1967. М.

Ннститут геологии

Академии наук Эстонской ССР
Поступила в редакцию 20/I 1976 

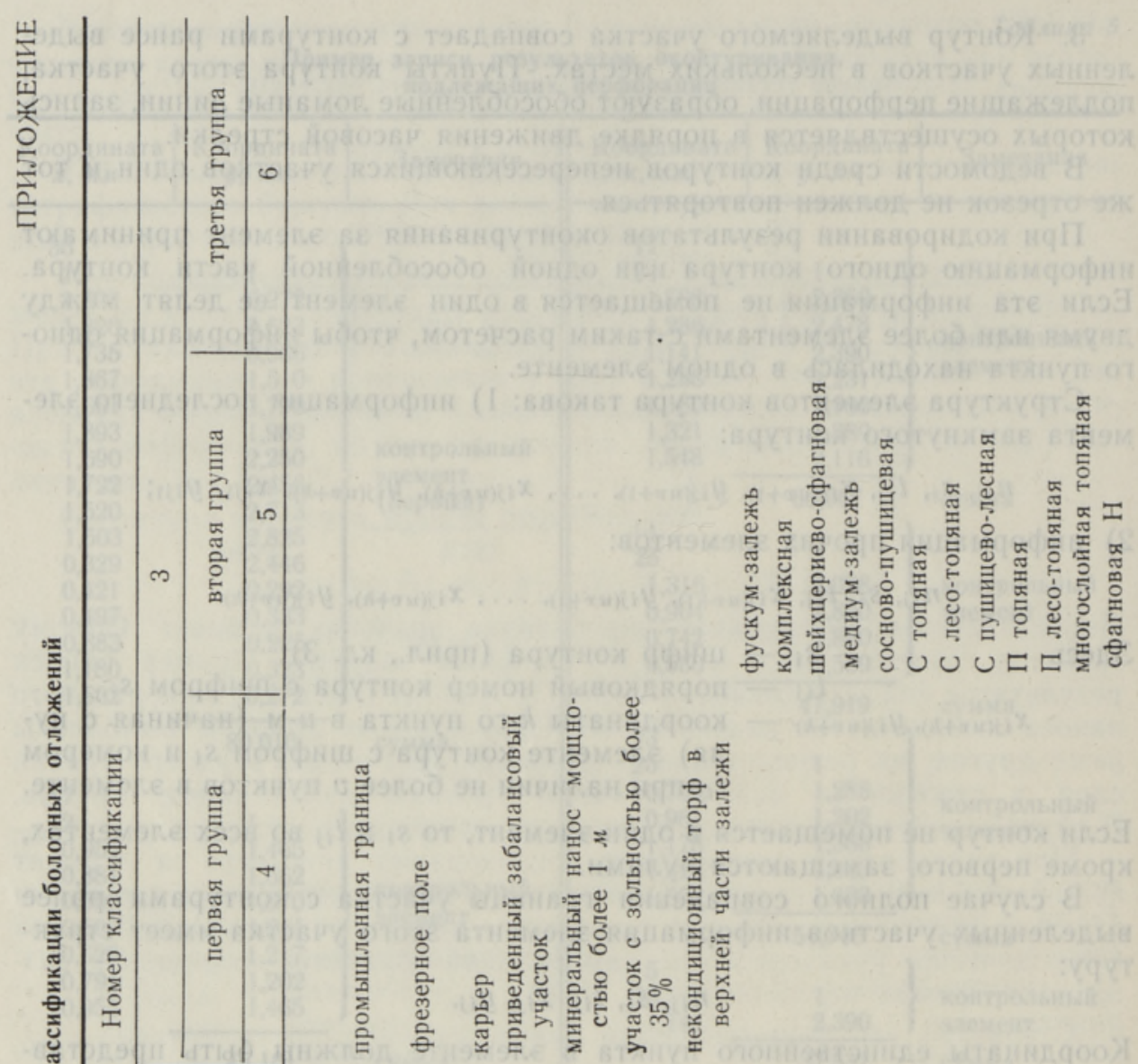

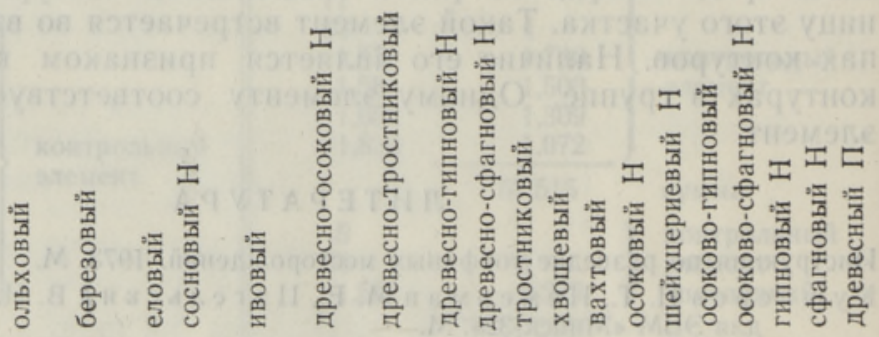

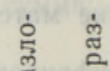

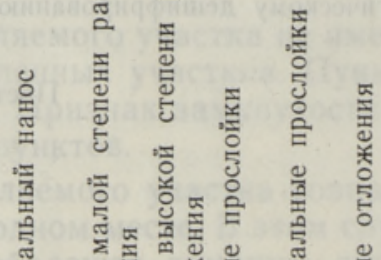

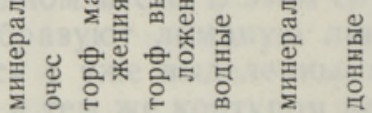



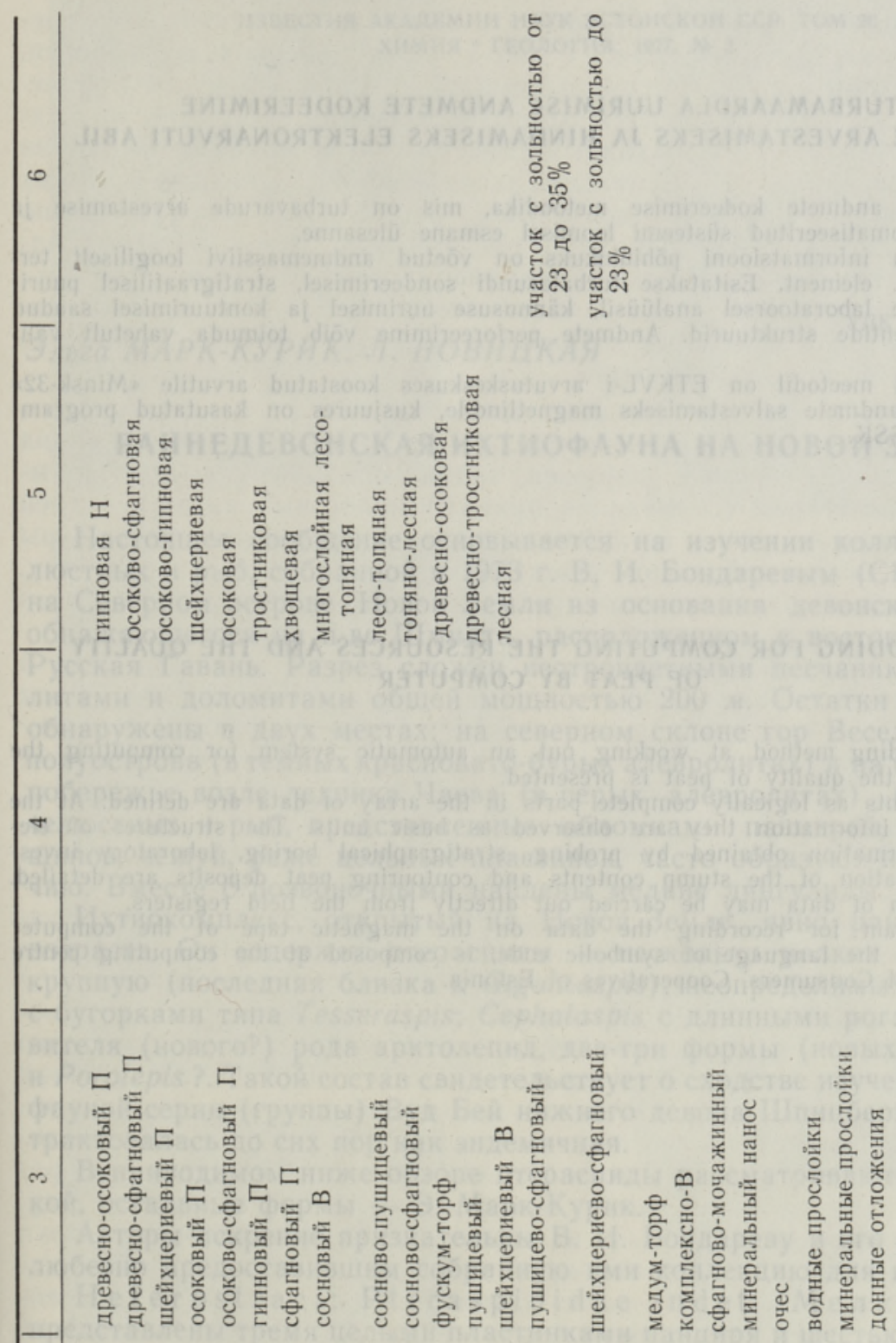

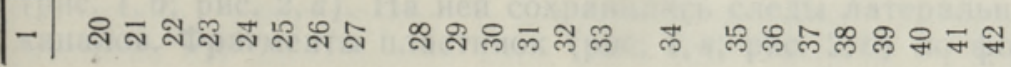




\section{K. ORGLA}

\section{TURBAMAARDLA UURIMISE ANDMETE KODEERIMINE VARUDE ARVESTAMISEKS JA HINDAMISEKS ELEKTRONARVUTI ABIL}

Esitatakse andmete kodeerimise metoodika, mis on turbavarude arvestamise ja hindamise automatiseeritud süsteemi loomisel esmane ülesanne.

Töödeldava informatsiooni pōhiühikuks on võetud andmemassiivi loogiliselt terviklik osa, nn. element. Esitatakse turbalasundi sondeerimisel, stratigraafilisel puurimisel, proovide laboratoorsel analüüsil, kännususe uurimisel ja kontuurimisel saadud andmete elementide struktuurid. Andmete perforeerimine võib toimuda vahetult välimaterjalidest.

Kirjeldatud meetodil on ETKVL-i arvutuskeskuses koostatud arvutile «Minsk-32» programm algandmete salvestamiseks magnetlindile, kusjuures on kasutatud programmeerimiskeelt SSK.

K. ORGLA

\section{DATA CODING FOR COMPUTING THE RESOURCES AND THE QUALITY OF PEAT BY COMPUTER}

A data coding method at working out an automatic system for computing the resources and the quality of peat is presented.

The elements as logically complete parts in the array of data are defined. At the processing of information they are observed as basic units. The structures of elements of information obtained by probing, stratigraphical boring, laboratory investigation, estimation of the stump contents and contouring peat deposits are detailed. The perforation of data may be carried out directly from the field registers.

The program for recording the data on the magnetic tape of the computer "Minsk-32", in the language of symbolic code, is composed at the computing centre of the Union of Consumers' Cooperatives of Estonia. 